\title{
On the optimality of planar and geometric approximation schemes
}

\author{
Dániel Marx \\ Institut für Informatik, \\ Humboldt-Universität zu Berlin, \\ dmarx@informatik.hu-berlin.de
}

\begin{abstract}
We show for several planar and geometric problems that the best known approximation schemes are essentially optimal with respect to the dependence on $\epsilon$. For example, we show that the $2^{O(1 / \epsilon)} \cdot n$ time approximation schemes for planar MAXIMUM INDEPENDENT SET and for TSP on a metric defined by a planar graph are essentially optimal: if there is a $\delta>0$ such that any of these problems admits a $2^{O\left((1 / \epsilon)^{1-\delta}\right)} n^{O(1)}$ time PTAS, then the Exponential Time Hypothesis (ETH) fails. It is known that MAXIMUM INDEPENDENT SET on unit disk graphs and the planar logic problems MPSAT, TMIN, TMAX admit $n^{O(1 / \epsilon)}$ time approximation schemes. We show that they are optimal in the sense that if there is a $\delta>0$ such that any of these problems admits a $2^{(1 / \epsilon)^{O(1)}} n^{O\left((1 / \epsilon)^{1-\delta}\right)}$ time PTAS, then ETH fails.
\end{abstract}

\section{Introduction}

Many classical graph-theoretic problems admit polynomial-time approximation schemes (PTAS) when restricted to planar graphs. For example, in planar graphs a $(1+\epsilon)$-approximation for MAXIMUM INDEPENDENT SET, Minimum Vertex Cover, Minimum Dominating SET [7], and TSP [19] can be computed in time $2^{O(1 / \epsilon)} \cdot n$. Planar graph problems and planar geometric problems share some structural similarities, hence two-dimensional geometric problems often have PTAS's. For example, given a set of unit disks in the plane, a $(1+\epsilon)$-approximation of the maximum independent set can be found in time $n^{O(1 / \epsilon)}$ [15].

Improving the dependence of the running time on $\epsilon$ is an obvious goal and there is a history of such improvements in the literature. Arora [5] presented an $n^{O(1 / \epsilon)}$ time PTAS for Euclidean TSP, which was improved to $n \cdot \log O(1 / \epsilon) n$ in the journal version of the paper [6]. Later, Rao and Smith [26] gave a PTAS with running time further improved to $2^{O((1 / \epsilon) \log (1 / \epsilon))} n+O(n \log n)$. Thus, given a PTAS, it seems worth investigating whether improvements in the dependence on $\epsilon$ are possible or we have reached the optimum PTAS. For planar graphs, an exact solution of MAXIMUM INDEPENDENT SET can be found in time $2^{O(\sqrt{n})}$ [2] (instead of the trivial $\left.2^{O(n)}\right)$; this might raise the hope that a $2^{O(\sqrt{1 / \epsilon})} \cdot n^{O(1)}$ PTAS exists. Finding a set of $k$ independent unit disks can be done in time $n^{O(\sqrt{k})}$ [3] (instead of the trivial $\left.n^{O(k)}\right)$, hence an $n^{O(\sqrt{1 / \epsilon})}$ time PTAS would not be completely surprising. The main contribution of the paper is proving almost-tight lower bounds for planar and geometric problems: we show that the known approximation schemes for these problems are essentially optimal with respect to the dependence on $\epsilon$ in the running time. To prove these lower bounds, we assume the Exponential Time Hypothesis (ETH): we assume that $n$-variable 3SAT cannot be solved in time $2^{o(n)}$. The Sparsification Lemma of Impagliazzo, Paturi, and Zane [16] states that this assumption is equivalent to the (seemingly stronger) assumption that there is no algorithm that solves 3SAT in $2^{o(n)}$, where $n$ is the size of the instance. We remark that instead of assuming ETH, the results in this paper follow from the weaker assumption that $m$-clause 3SAT cannot be solved in time $2^{O\left(m^{1-\delta}\right)}$ for any $\delta>0$.

The following theorem states our lower bounds for five problems that (with the exception of the last) are known to admit approximation schemes with running time $2^{O(1 / \epsilon)} \cdot n$.

Theorem 1.1. Assuming ETH, there is no $\delta>0$ such that $a 2^{O\left((1 / \epsilon)^{1-\delta}\right)} n^{O(1)}$ time PTAS exists for

- MAXIMUm IndePEndent SET on planar graphs,

- Minimum Vertex Cover on planar graphs,

- Minimum Dominating Set on planar graphs,

- TSP with a metric defined by an unweighted planar graph.

- Minimum Vertex Cover for unit disks or unit squares. 
The problems considered in the following theorem admit approximation schemes with running time $n^{O(1 / \epsilon)}$; we show that $1 / \epsilon$ in the exponent of $n$ cannot be replaced by $(1 / \epsilon)^{1-\delta}$ for any $\delta>0$. The statement of the theorem is slightly stronger: the lower bound on the exponent holds even if we allow an exponential function of $1 / \epsilon$ as a multiplier:

Theorem 1.2. Assuming ETH, there is no $\delta>0$ such that $a 2^{(1 / \epsilon)^{O(1)}} \cdot n^{O\left((1 / \epsilon)^{(1-\delta)}\right)}$ time PTAS exists for

- MPSAT for planar formulas,

- TMIN for planar formulas,

- TMAX for planar formulas,

- Maximum IndePendent Set for unit disks or squares.

- Minimum Dominating Set for unit disks or squares.

Ideally, for these problems we would like to have a tight result that rules out the possibility of a PTAS with running time $f(\epsilon) n^{o(1 / \epsilon)}$, for any function $f(\epsilon)$. For example, Theorem 1.2 does not rule out the possibility of a PTAS with running time, say, $2^{2^{2^{1 / \epsilon}}} n^{\log \log (1 / \epsilon)}$ — such a PTAS could be considered as a theoretical improvement over $n^{O(1 / \epsilon)}$ time. Without going into details, we mention that it is possible to prove the weaker result that no $f(\epsilon) n^{o(\sqrt{1 / \epsilon})}$ PTAS exists for any function $f(\epsilon)$ for these problems. Theorems 1.1 and 1.2 are not tight because we are using the almost-linear size PCP construction of Dinur [12]. Replacing this with a linear-size PCP would result in tight lower bounds, but currently such a construction is not in sight.

A simple observation gives us non-tight lower bounds on the dependence on $\epsilon$. Since MAXIMUM INDEPENDENT SET is NP-hard for planar graphs, there is a polynomialtime algorithm that turns a 3SAT instance of size $n$ into an equivalent instance of MAXIMUM INDEPENDENT SET on a planar graph of size $n^{c}$, for some constant $c$. By setting $\epsilon:=1 /\left(n^{c}+1\right)$, a $(1+\epsilon)$-approximation algorithm can solve the constructed instance of MAXIMUM INDEPENDENT SET exactly. Therefore, no PTAS with running time $2^{o\left((1 / \epsilon)^{1 / c}\right)} n^{O(1)}$ can exist: otherwise it would be able to solve 3SAT in time $2^{o(n)}$, contradicting ETH. By observing that $c=2$ in the known reductions from 3SAT to planar MAXIMUM INDEPENDENT SET, we obtain a lower bound of $2^{\sqrt{1 / \epsilon}} \cdot n^{O(1)}$. However, this argument cannot be used to prove stronger lower bounds: as planar MAXIMUM INDEPENDENT SET can be solved in time $2^{O(\sqrt{n})}$ [2], a reduction with $c<2$ would mean that 3SAT can be solved in time $2^{o(n)}$. Therefore, new techniques are required for the almost-tight bounds of Theorems 1.1.
The situation is similar in the case of MAXIMUM INDEPENDENT SET for unit disks. The W[1]-hardness proof of [24] together with a result of [11] implies that there is a constant $c>0$ such that there is no $f(k) n^{o\left(k^{1 / c}\right)}$ time algorithm for finding a set of $k$ independent disks, unless ETH fails. This means (by a simple argument of Bazgan [8] and Cesati and Trevisan [10]) that no PTAS with running time $f(\epsilon) n^{o\left((1 / \epsilon)^{1 / c}\right)}$ can exist. However, the problem can be solved in time $n^{O(\sqrt{k})}$ [3], thus $c \geq 2$, which means that we cannot prove a lower bound stronger than $f(\epsilon) n^{O(\sqrt{1 / \epsilon})}$ with this argument.

We get around these difficulties by using the fact that, assuming ETH, not only 3SAT cannot be solved in time $2^{o(n)}$, but the optimization version MAX3SAT cannot be approximated to some constant factor in time $2^{O\left(n^{1-\delta}\right)}$ for any $\delta>0$. This is a consequence of the almost-linear size PCP of Dinur [12]. The lower bounds for the planar problems are obtained by a reduction that is approximation preserving in a weak way: a fast PTAS for the planar problem would imply a fast constant-factor approximation for MAX3SAT. Note that this reduction is somewhat unusual, since a problem without a PTAS is reduced to a problem that admits a PTAS.

Previously, the literature focused mostly on the PTAS vs. EPTAS question $[10,9,13,24,23,25]$. An efficient PTAS (EPTAS) is an approximation scheme with running time $f(\epsilon) n^{O(1)}$ for some function $f$. These papers showed, for problems that were known to admit approximation schemes, that no EPTAS exists (under the standard parameterized complexity assumption W[1] $\neq$ FPT). These results can be turned into non-tight lower bounds on the exponent of $n$. In [4], almost-tight lower bounds were obtained for the string matching problem CLOSEST SUBSTRING; this is the only previous result that we are aware of where almost-tight bounds were established. Our result give almost-tight bounds for most of the problems considered in $[10,9,13,24]$. Furthermore, we also give almosttight lower bounds for problems that admit EPTASs (Theorem 1.1); to our knowledge, these are the first results of this type.

In Section 2, we introduce the planar problem MATRIX TILING. This problem admits an $n^{O(1 / \epsilon)}$ time PTAS and a special case of the problem admits a $2^{O(1 / \epsilon)} n$ time PTAS. We show that these approximation schemes are optimal in the sense of Theorem 1.1 and 1.2. The lower bounds of Theorem 1.1 and 1.2 are obtained by reducing MATRIX TILING (or its special case) to the various problems (Sections 36). MATRIX TILING was defined with such reductions in mind, thus the reductions are fairly straightforward if we can construct the problem-specific gadgets. It is likely that reduction from MATRIX TILING can be used to prove lower bounds for many other planar and geometric problems. 


\section{The Matrix Tiling problem}

The problem MATRIX TILING plays a central role in the paper: we prove lower bounds on the efficiency of the approximation schemes of MATRIX TILING and these lower bounds are transfered to other problems by appropriate reductions. We denote by $Z_{D}$ the set $\{0,1, \ldots, D-1\}$ throughout the paper.

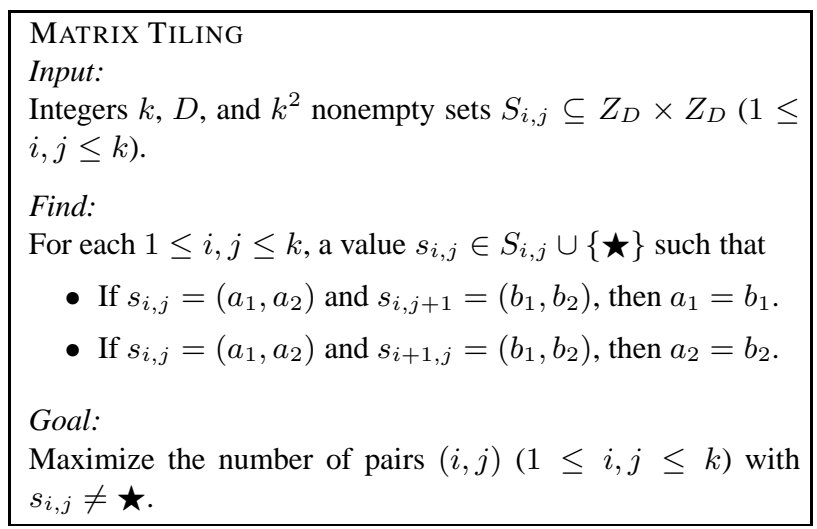

We think of the values $s_{i, j}$ of a solution as being elements of a matrix, hence we use the expressions row, column, and cell with the obvious meaning. Observe that the optimum is always at least $k^{2} / 4$ : if $i$ and $j$ are both odd, then let $s_{i, j}$ be an arbitrary element of $S_{i, j}$, otherwise let $s_{i, j}=\star$. (Here we use that $S_{i, j}$ is required to be nonempty.) This observation will be useful when reducing MATRIX TILING to other problems. The size of the input instance can be bounded by $O\left(k^{2} D^{2}\right)$.

The following lemma gives a reduction from MAX3SAT to MATRIX TILING. The reduction is approximationpreserving in a certain weak sense, which allows use to use a PTAS for MATRIX TILING to solve MAX3SAT. A formula with $m$ clauses is $\alpha$-satisfiable if there is an assignment that satisfies at least $\alpha m$ clauses.

Lemma 2.1. There is an algorithm with running time polynomial in the size of the output that, given a 3SAT formula $\phi$ having $m$ clauses and an integer $k$, constructs an instance of MATRIX TILING with parameters $k$ and $D:=3^{\lceil m / k\rceil}$ such that for every $1>\alpha>0$,

- if $\phi$ is satisfiable, then the optimum of MATRIX TILING is $k^{2}$,

- if $\phi$ is not $\alpha$-satisfiable, then the optimum of MATRIX TILING is at most $k^{2}-k(1-\alpha) / 2+1$.

Proof. Let us associate a variable $y_{i} \in\{1,2,3\}$ with each clause of $\phi$. The intended meaning of $y_{i}=\ell$ is that the $i$-th clause is satisfied by its $\ell$-th literal. To enforce this interpretation, we construct a set of constraints: if the $\ell_{1}$-th literal of the $i_{1}$-th clause is the negation of the $\ell_{2}$-th literal of the $i_{2}$-th clause, then the constraint $\left(y_{i_{1}} \neq \ell_{1} \vee y_{i_{2}} \neq \ell_{2}\right)$ is added to the set. It is clear that a subset $C_{0}$ of clauses is satisfiable if and only if there is an assignment to the corresponding $\left|C_{0}\right|$ variables that satisfies all the constraints induced by $C_{0}$.

We want to partition the $y_{i}$ 's into $k$ blocks $B_{1}, \ldots$, $B_{k}$ of equal size, but $m / k$ is not necessarily integer. Let $m^{\prime}:=k\lceil m / k\rceil$ and let us add $m^{\prime}-m \leq m^{\prime} / k$ new dummy variables that do not appear in any of the constraints. Set $D:=3^{m^{\prime} / k}$; for each block of $m^{\prime} / k$ variables, we can fix a bijection between $Z_{D}$ and the possible assignments of the variables in the block. We construct an instance of MATRIX TILING with parameters $k$ and $D$ where the sets $S_{i, j}$ are defined as follows. We say that a partial assignment $\gamma$ of the variables $y_{i}$ is compatible, if there is no constraint $\left(y_{i_{1}} \neq \ell_{1} \vee y_{i_{2}} \neq \ell_{2}\right)$ with $\gamma\left(y_{i_{1}}\right)=\ell_{1}$ and $\gamma\left(y_{i_{2}}\right)=\ell_{2}$. If $i=j$, then $S_{i, j}$ contains those pairs $(t, t)$ where $t \in Z_{D}$ corresponds to a compatible assignment of block $B_{i}$. For $i \neq j$, consider a pair $\left(v_{i}, v_{j}\right) \in Z_{D} \times Z_{D}$. Let $\gamma_{i}$ (resp., $\gamma_{j}$ ) be the assignment of block $B_{i}$ (resp., $B_{j}$ ) that corresponds to $v_{i}$ (resp., $\left.v_{j}\right)$. The pair $\left(v_{i}, v_{j}\right)$ is in $S_{i, j}$ if and only if $\gamma_{i}$ and $\gamma_{j}$ together form a compatible assignment of $B_{i} \cup B_{j}$. If a set $S_{i, j}$ is empty, then $\phi$ is unsatisfiable, since there is no compatible assignment for some pair of blocks. In this case, we can output an arbitrary instance with optimum $k^{2} / 4$. This completes the description of the constructed instance of MATRIX TILING.

If $\phi$ is satisfiable, then there is an assignment $\gamma$ of the $y_{i}$ 's that satisfies all the constraints we defined. For each $1 \leq i, j \leq k$, let $v_{i} \in Z_{D}$ be the value corresponding to assignment $\gamma$ restricted to block $B_{i}$. Set $s_{i, j}=\left(v_{i}, v_{j}\right)$. Assignment $\gamma$ satisfies all the constraints; in particular, it satisfies all the constraints induced by the variables in $B_{i} \cup$ $B_{j}$, implying $\left(v_{i}, v_{j}\right) \in S_{i, j}$. Thus we obtain a solution with value $k^{2}$.

For the second part, suppose that MATRIX TILING has a solution with value at least $k^{2}-k(1-\alpha) / 2+1$, i.e., the number of $\star$ 's is at most $k(1-\alpha) / 2-1$. Let us call a block $B_{i}$ bad if the $i$-th row or the $i$-th column contains at least one $\star$. As a $\star$ can make at most 2 blocks bad, there are at most $(1-\alpha) k-2$ bad blocks. Let us call a variable $y_{t}$ and (if $y_{t}$ is not a dummy variable) the corresponding clause of $\phi$ good, if $y_{t}$ does not belong to a bad block; clearly, there are at least $\left(m^{\prime} / k\right)(k-(1-\alpha) k+2)=\alpha m^{\prime}+2 m^{\prime} / k$ good variables. Less than $m^{\prime} / k$ of the good variables are dummy variables, hence we have at least $\alpha m$ good clauses. We claim that there is an assignment of $\phi$ satisfying all the good clauses, contradicting the assumption that $\phi$ is not $\alpha$ satisfiable. If block $B_{r}$ is not bad, then there is a value $v_{r}$ such that the first component of each $s_{r, j}(1 \leq j \leq k)$ is $v_{r}$. Similarly, there is a value $v_{r}^{\prime}$ such that the second component of each $s_{i, r}(1 \leq i \leq k)$ is $v_{r}^{\prime}$. Since $\left(v_{r}, v_{r}^{\prime}\right) \in S_{r, r}$, we get that $v_{r}=v_{r}^{\prime}$. The value $v_{r}$ defines an assignment of the variables $y_{t}$ in block $v_{r}$; this way, we obtain a value for 
each good variable $y_{t}$. In a natural way, we can construct an assignment of $\phi$ corresponding to the values of the good variables: $y_{t}=\ell$ means that the $t$-th clause is satisfied by its $\ell$-th literal, hence it defines the value of one variable of $\phi$; if a variable $x_{i}$ of $\phi$ is not assigned a value this way, then we can assign an arbitrary value to it. This assignment is well defined: if $y_{t_{1}}=\ell_{1}$ and $y_{t_{2}}=\ell_{2}$ force a variable to different values, then there is a constraint $\left(y_{t_{1}} \neq \ell_{1} \vee y_{t_{2}} \neq \ell_{2}\right)$. Suppose that $y_{t_{1}} \in B_{r_{1}}, y_{t_{2}} \in B_{r_{2}}$; this means that the assignment of $B_{r_{1}}$ corresponding to $v_{r_{1}}$ is not compatible with the assignment of $B_{r_{2}}$ corresponding to $v_{r_{2}}$, contradicting the fact that $s_{r_{1}, r_{2}}=\left(v_{r_{1}}, v_{r_{2}}\right) \in S_{r_{1}, r_{2}}$. Therefore, the constructed assignment satisfies every good clause, i.e., $\phi$ is $\alpha$-satisfiable.

Using standard layering techniques, it can be shown that MATRIX TILING admits a PTAS with running time $n^{O(1 / \epsilon)}$. In Theorem 2.3, we show that the $1 / \epsilon$ in the exponent of $n$ is essentially optimal. The lower bound is obtained from known hardness results on MAX3SAT.

The Sparsification Lemma of Impagliazzo, Paturi, and Zane [16] implies that ETH is equivalent to the assumption that $m$-clause 3SAT cannot be solved in time $2^{o(m)}$. The almost-linear size PCP of Dinur can be used to turn a formula $\phi$ with $m$ clauses into a formula $\psi$ with $m^{\prime}:=$ $m \log ^{O(1)} m$ clauses such that if $\phi$ is satisfiable, then $\psi$ is satisfiable and if $\phi$ is unsatisfiable, then $\psi$ is not $\alpha$ satisfiable for some constant $\alpha<1$. Therefore, the running time of an algorithm distinguishing between these two cases cannot be $2^{o(m)}$, which means that it cannot be $2^{O\left(m^{\prime(1-\delta)}\right)}$ for any $\delta>0$.

Lemma 2.2. There is a constant $1>\alpha>0$ such that if there is an algorithm that can distinguish between satisfiable and not $\alpha$-satisfiable 3SAT formulas in time $2^{O\left(m^{1-\delta}\right)}$ for some constant $\delta>0$ (where $m$ is the number of clauses), then ETH fails.

Theorem 2.3. If there are constants $\delta, d>0$ such that MATRIX TILING has a PTAS with running time $2^{O(1 / \epsilon)^{d}}$. $n^{O(1 / \epsilon)^{1-\delta}}$, then ETH fails.

Proof. Let $\phi$ be a 3SAT formula with $m$ clauses. Set $k:=$ $\left\lceil m^{1 /(2 d+1)}\right\rceil \leq m$. Let us use the algorithm of Lemma 2.1 to construct an instance of MATRIX TILING from $\phi$ with this value of $k$. Set $\epsilon:=(1-\alpha) /(2 k)-1 / k^{2}=\Theta(1 / k)$, where $\alpha$ is the universal constant in Theorem 2.2 (we assume that $m$, and hence $k$, is sufficiently large that $\epsilon$ is positive.) If $\phi$ is satisfiable, then the optimum of MATRIX TILING is $k^{2}$. On the other hand, if $\phi$ is not $\alpha$-satisfiable, then the optimum is at most $k^{2}-k(1-\alpha) / 2+1$. Since $k^{2} /(1+\epsilon)>k^{2}(1-\epsilon)=k^{2}-k(1-\alpha) / 2+1, \mathrm{a}$ $(1+\epsilon)$-approximation algorithm can distinguish between the two cases. If there is a PTAS with running time
$2^{O(1 / \epsilon)^{d}} \cdot n^{O(1 / \epsilon)^{(1-\delta)}}$, then the two cases can be distinguished in time

$$
\begin{aligned}
& 2^{O\left(k^{d}\right)} \cdot\left(k^{2} \cdot D^{2}\right)^{O(k)^{1-\delta}} \\
& =\exp \left(O\left(k^{d}\right)+(O(\log k)+O(m / k)) \cdot O(k)^{1-\delta}\right) \\
& =\exp \left(O\left(m^{d /(2 d+1)}\right)+o(k)+O\left(m / k^{\delta}\right)\right) \\
& \quad=\exp \left(o(m)+O\left(m^{1-\delta /(2 d+1)}\right)\right)=2^{o(m)},
\end{aligned}
$$

which, by Lemma 2.2, contradicts ETH.

Let us investigate the special case of MATRIX TILING with $D=2$. It can be shown that this special case admits a PTAS with running time $2^{O(1 / \epsilon)} n$ and, as we shall see in Theorem 2.6, this is essentially optimal. The lower bounds in Theorem 1.1 are obtained by reductions from this special case. When reducing from this special case, $\left|S_{i, j}\right| \leq 4$ implies that a "gadget" representing some $S_{i, j}$ has to have only a constant number of states. Therefore, the gadget construction can be simpler and the reduction can be done to a wider range of problems.

If $D=2$, then we are not able to reduce MAX3SAT to MATRIX TILING as in Lemma 2.1. Instead, we reduce MAX2SAT. We say that a 2SAT formula is simple, if it does not contain unsatisfiable clauses or duplicated clauses.

Lemma 2.4. There is a polynomial-time algorithm that, given a simple 2SAT formula $\phi$ with $m$ clauses where each variables appears in at most $d$ clauses, constructs an instance of MATRIX TILING with parameters $k:=O\left(d^{2} m\right)$ and $D:=2$ such that if $t$ is the minimum number of unsatisfied clauses in $\phi$, then the optimum of the constructed instance is $k^{2}-t$.

Proof. Let $x_{1}, \ldots, x_{p}$ be an ordering of the variables of $\phi$ $(p \leq 2 m)$. By adding new variables, we obtain a longer sequence $X$ of variables. Let $x_{i_{1}}, \ldots, x_{i_{d^{\prime}}}$ be those variables that appear together with $x_{i}$ in some clause $\left(d^{\prime} \leq d\right)$. Set $z:=4 d$. We replace $x_{i}$ with a sequence of $2 z d^{\prime}+1$ variables, called the segment of $x_{i}$. This segment contains $x_{i}$ and $2 z d^{\prime}$ new variables $x_{i_{s}, i, \ell}\left(1 \leq s \leq d^{\prime}, 1 \leq \ell \leq 2 z\right)$. The variables in the segment are ordered in such a way that $x_{i_{s}, i, \ell}$ is before $x_{i}$ for $1 \leq \ell \leq z$ and it is after $x_{i}$ for $z+1 \leq \ell \leq 2 z$. Replacing every $x_{i}$ with the corresponding segment of $2 z d^{\prime}+1$ variables gives a sequence $\mathrm{X}$ of $k \leq p(2 z d+1)=O\left(d^{2} m\right)$ variables. For each new variable $x_{i_{s}, i, \ell}$, we say that it is a copy of $x_{i_{s}}$; each variable has at most $2 z d$ copies (we do not consider a variable $x_{i}$ to be a copy of itself). If a variable is a copy of $x_{i}$ or it is $x_{i}$ itself, then we say that the variable represents $x_{i}$. We construct an instance of MATRIX TILING with parameters $k$ and $D=2$ where the rows and the columns are indexed by the $k$ variables in $X$. The sets $S_{i, j}$ are defined the following way. If there is a clause $\left(x_{i} \vee x_{j}\right)$, then the set in 
row $x_{i}$ and column $x_{j}$ is $\{(0,1),(1,0),(1,1)\}$. We consider a clause as an ordered pair of literals, hence clause $\left(x_{i} \vee x_{j}\right)$ does not influence the set in row $x_{j}$ of column $x_{i}$. We proceed similarly for clauses containing negations: in this case, the set $S_{i, j}$ contains the three pairs corresponding to the satisfying assignments of the clause. Every other set is $\{(0,0),(0,1),(1,0),(1,1)\}$, unless the row and the column indices represent the same variable $x_{i}$, in which case the set is $\{(0,0),(1,1)\}$.

Assume that $\phi$ has an assignment $\gamma$ that satisfies all but $t$ of the clauses. We define the value of $s_{i, j}$ the following way. If the $i$-th variable in sequence $X$ represents $x_{i_{0}}$ and the $j$-th variable represents $x_{j_{0}}$, then $s_{i, j}=\left(\gamma\left(x_{i_{0}}\right), \gamma\left(x_{j_{0}}\right)\right)$. This pair is in $S_{i, j}$ unless $S_{i, j}$ corresponds to a clause not satisfied by $\gamma$. That is, the $i$-th (resp., $j$-th) variable of the sequence is $x_{i_{0}}$ (resp., $x_{j_{0}}$ ) itself (not a copy) and there is an unsatisfied clause where the first (resp., second) variable is $x_{i_{0}}$ (resp., $x_{j_{0}}$ ). If the pair is not in $S_{i, j}$, then we set $s_{i, j}=\star$; clearly, the number of $\star$ 's is at most $t$.

Assume now that there is a solution with value at least $k^{2}-t$. A variable of the sequence $X$ is bad if the row or the column corresponding to the variable contains at least one $\star$; otherwise let us call it good. If the $i$-th variable of the sequence $X$ is good, then let us associate to this variable the value that appears as the first component of each pair in the $i$-th row. Because of the way the set $S_{i, i}$ is defined, this value is the same as the value that appears as the second component of each pair in the $i$-th column. If the $i$-th and the $j$-th variables of $X$ are both good and they represent the same variable, then the same value is associated to them: set $S_{i, j}=\{(0,0),(1,1)\}$ ensures this. A variable of $\phi$ is spoiled if at least $z$ of its copies are bad. We construct an assignment $\gamma$ of $\phi$ the following way: if $x_{i}$ is spoiled, then we set $\gamma\left(x_{i}\right)$ arbitrarily, otherwise let $\gamma\left(x_{i}\right)$ be the common value associated with the good copies of $x_{i}$.

We claim that at most $t$ clauses of $\phi$ are not satisfied by $\gamma$. For each clause, we define a set of cells called the cross of the clause. Let $x_{i}, x_{j}$ be the two variables of the clause. The cross of the clause contains those cells of row $x_{i}$ whose column belongs to the segment of $x_{j}$ and contains those cells of column $x_{j}$ whose row belongs to the segment of $x_{i}$. It is easy to see that the crosses for different clauses are disjoint (here we use the assumption that there are no duplicated clauses in $\phi$ ). We claim that for every unsatisfied clause, at least one of the following is true: (1) the cell in row $x_{i}$ of column $x_{j}$ contains a $\star$, (2) the clause has a spoiled variable, (3) the cross of the clause contains at least two $\star$ 's. Consider a clause such that none of (1)-(3) hold. Since $x_{i}$ is not spoiled, there is an $1 \leq \ell_{i, 1} \leq z$ such that variable $x_{i, j, \ell_{i, 1}}$ is good and there is a $z+1 \leq \ell_{i, 2} \leq 2 z$ such that variable $x_{i, j, \ell_{i, 2}}$ is good. If there is no $\star$ in row $x_{i}$ between columns $x_{i, j, \ell_{i, 1}}$ and $x_{j}$, then the first component of row $x_{i}$ of column $x_{j}$ is $\gamma\left(x_{i}\right)$. Similarly, if there is no $\star$ in row $x_{i}$ between columns $x_{i}$ and $x_{i, j, \ell_{i, 2}}$, then the first component of row $x_{i}$ of column $x_{j}$ is again $\gamma\left(x_{i}\right)$. As neither (1) nor (3) holds for the clause, at least one of these two statements has to be true. A similar argument shows that the second component of row $x_{i}$ of column $x_{j}$ is $\gamma\left(x_{j}\right)$. Therefore, the fact that the pair $\left(\gamma\left(x_{i}\right), \gamma\left(x_{j}\right)\right)$ appears in the corresponding cell implies that the clause is satisfied by $\gamma$.

Let $t_{0}$ be the number of clauses for which (1) is true. Observe that the corresponding $t_{0} \star$ 's do not influence whether (2) or (3) are true for the other clauses. Therefore, it is sufficient to investigate the effect of the remaining $t-t_{0} \star$ 's. $\mathrm{A} \star$ can make at most 2 variables of the sequence $X$ bad, hence there are at most $2\left(t-t_{0}\right) / z$ spoiled variables in $\phi$. Therefore, (2) is true for at most $d \cdot 2\left(t-t_{0}\right) / z \leq\left(t-t_{0}\right) / 2$ clauses. As the crosses are disjoint, at most $\left(t-t_{0}\right) / 2$ of them can contain at least two $\star$ 's. Therefore, the total number of unsatisfied clauses is at most $t_{0}+\left(t-t_{0}\right) / 2+\left(t-t_{0}\right) / 2=t$.

Combining Lemma 2.2 with standard reductions, we get

Lemma 2.5. There are constants $1>\alpha_{1}>\alpha_{2}>0$ such that if there is an algorithm that can distinguish between $\alpha_{1}$-satisfiable and not $\alpha_{2}$-satisfiable 2SAT formulas in time $2^{O\left(m^{1-\delta}\right)}$ for some constant $\delta>0$ (where $m$ is the number of clauses), then ETH fails. Furthermore, we can assume that the formula is simple and a variable appears at most $d$ times for some constant $d>0$.

Theorem 2.6. If there is a $\delta>0$ such that MATRIX TILING has a PTAS with running time $2^{O(1 / \epsilon)^{1-\delta}} \cdot n^{O(1)}$ in the special case $D=2$, then ETH fails.

Proof. Let $\phi$ be a 3SAT formula with $m$ clauses. Let us use the algorithm of Lemma 2.4 to construct an instance of MATRIX TILING with $k=O\left(d^{2} m\right)$ and $D=2$. If $\phi$ is $\alpha_{1}$ satisfiable, then the optimum of the constructed instance of MATRIX TILING is at least $k^{2}-\left(1-\alpha_{1}\right) m$, while if $\phi$ is not $\alpha_{2}$-satisfiable, then the optimum is less than $k^{2}-\left(1-\alpha_{2}\right) m$. This means that by setting $\epsilon:=\left(\alpha_{1}-\alpha_{2}\right) m /\left(4 k^{2}\right)=$ $O(1 / k)$, a $(1+\epsilon)$-approximation algorithm can distinguish between the two cases. Therefore, if the assumed PTAS exists, then this can be done in time

$$
\begin{array}{r}
2^{O(1 / \epsilon)^{1-\delta}} \cdot n^{O(1)}=2^{O(k)^{1-\delta}} \cdot k^{O(1)}=2^{O(m)^{1-\delta}+O(\log k)} \\
=2^{O\left(m^{1-\delta}\right)}
\end{array}
$$

which, by Lemma 2.5, contradicts ETH.

Having proved the lower bounds of Theorems 2.3 and 2.6, we transfer these bounds to other optimization problems by means of an L-reduction.

Definition 2.7. Let $A$ and $B$ be optimization problems and $c_{A}$ and $c_{B}$ their respective cost functions. A pair of 
logspace-computable functions $R$ and $S$ is an L-reduction if all of the following conditions are met:

- if $x$ is an instance of problem $A$, then $R(x)$ is an instance of problem $B$,

- if $y$ is a solution to $R(x)$, then $S(y)$ is a solution to $x$,

- there exists a constant $\alpha>0$ such that $\mathrm{OPT}(R(x)) \leq$ $\alpha \operatorname{OPT}(x)$.

- there exists a constant $\beta>0$ such that $\mid \mathrm{OPT}(x)-$ $c_{A}(S(y))|\leq \beta| \mathrm{OPT}(R(x))-c_{B}(y) \mid$.

It is easy to see that the bounds of Theorems 2.3 and 2.6 remain valid under L-reductions:

Lemma 2.8. (1) If there is an L-reduction from MATRIX TILING to Problem $X$, then there are no $d, \delta>0$ such that Problem $X$ admits a PTAS with running time $2^{O\left((1 / \epsilon)^{d}\right)} n^{O\left((1 / \epsilon)^{1-\delta}\right)}$, unless ETH fails.

(2) If there is an L-reduction from MATRIX TILING with $D=2$ to Problem $X$, then there is no $\delta>0$ such that Problem $X$ admits a PTAS with running time $2^{O\left((1 / \epsilon)^{1-\delta}\right)} n^{O(1)}$, unless ETH fails.

\section{Planar logic problems}

Khanna and Motwani [18] defined classes of optimization problems that admit polynomial-time approximation schemes. The problems are formulated using Boolean logical expressions. A formula in disjunctive normal form (DNF) is a disjunction of terms. A DNF is positive (resp., negative), if every literal is positive (resp., negated). The weight of an assignment is the number of variables that are set to true.

\begin{tabular}{|c|c|}
\hline $\begin{array}{l}\text { MPSAT } \\
\text { Input: } \\
\text { Find: } \\
\text { Goal: }\end{array}$ & $\begin{array}{l}\text { A collection } \mathcal{C}=\left\{\phi_{1}, \ldots, \phi_{n}\right\} \text { of DNFs. } \\
\text { An assignment } \gamma \text {. } \\
\text { Maximize the number of DNFs satisfied by } \gamma \text {. }\end{array}$ \\
\hline $\begin{array}{l}\text { TMIN } \\
\text { Input: } \\
\text { Find: } \\
\text { Goal: }\end{array}$ & $\begin{array}{l}\text { A collection } \mathcal{C}=\left\{\phi_{1}, \ldots, \phi_{n}\right\} \text { of positive DNFs. } \\
\text { An assignment } \gamma \text { that satisfies every DNF in } \mathcal{C} \text {. } \\
\text { Minimize the weight of } \gamma \text {. }\end{array}$ \\
\hline $\begin{array}{l}\text { TMAX } \\
\text { Input: } \\
\text { Find: } \\
\text { Goal: }\end{array}$ & $\begin{array}{l}\text { A collection } \mathcal{C}=\left\{\phi_{1}, \ldots, \phi_{n}\right\} \text { of negative DNFs. } \\
\text { An assignment } \gamma \text { that satisfies every DNF in } \mathcal{C} \text {. } \\
\text { Maximize the weight of } \gamma .\end{array}$ \\
\hline
\end{tabular}

These problems generalize many of the standard optimization problems: for example, MAX CUT can be reduced to MPSAT; MAXIMUM INDEPENDENT SET can be reduced to TMAX; and MINIMUM VERTEX COVER can be reduced to TMIN. (In all three reductions, we associate a

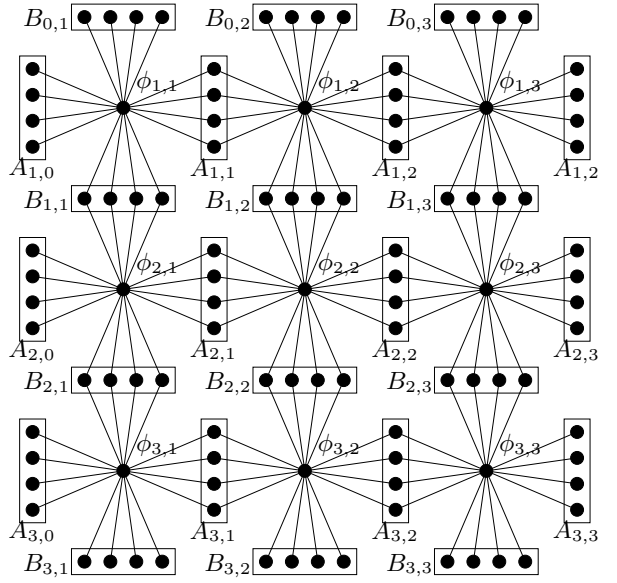

Figure 1. Structure of the instance constructed in Theorem $3.1(D=4, k=3)$.

variable with each vertex and a DNF with each edge.) Given an instance of the above problems, the incidence graph is a bipartite graph defined by associating a vertex to each variable and to each DNF, and by connecting each variable to every DNF where it appears. Khanna and Motwani [17] show that MPSAT, TMIN, TMAX all admit $n^{O(1 / \epsilon)}$ time PTAS's if the incidence graph is planar. Here we prove that these approximation schemes are essentially optimal:

Theorem 3.1. If there is a $\delta>0$ such that PLANAR MPSAT has a PTAS with running time $2^{(1 / \epsilon)^{O(1)}} \cdot n^{O(1 / \epsilon)^{1-\delta}}$, then ETH fails.

Proof. We present an L-reduction from MATRIX TILING to PLANAR MPSAT. The collection $\mathcal{C}$ consists of $k^{2}$ DNFs $\phi_{i, j}(1 \leq i, j \leq k)$ on $2 k(k+1) D$ variables. The variables are arranged into blocks $A_{i, j}(1 \leq i \leq k, 0 \leq j \leq k)$ and $B_{i, j}(0 \leq i \leq k, 1 \leq j \leq k)$, where each block contains $D$ variables. The variables in block $A_{i, j}$ (resp., $B_{i, j}$ ) will be denoted by $a_{i, j, s}$ (resp., $b_{i, j, s}$ ) for $s \in Z_{D}$. The DNF $\phi_{i, j}$ $(1 \leq i, j \leq t)$ contains variables only from blocks $A_{i, j-1}$, $A_{i, j}, B_{i-1, j}, B_{i, j}$. As shown in Figure 1, the incidence graph is planar. The formulas are defined as

$$
\begin{gathered}
\phi_{i, j}=\bigvee_{(x, y) \in S_{i, j}}\left(a_{i, j-1, x} \wedge a_{i, j, x} \wedge b_{i-1, j, y} \wedge b_{i, j, y}\right. \\
\left.\wedge \bigwedge_{x^{\prime} \neq x} \bar{a}_{i, j-1, x^{\prime}} \wedge \bigwedge_{x^{\prime} \neq x} \bar{a}_{i, j, x^{\prime}} \wedge \bigwedge_{y^{\prime} \neq y} \bar{b}_{i-1, j, y^{\prime}} \wedge \bigwedge_{y^{\prime} \neq y} \bar{b}_{i, j, y^{\prime}}\right) .
\end{gathered}
$$

This completes the description of the instance. We claim that the optimum of the constructed instance is the same as the optimum of MATRiX Tiling. Assume that MATRiX TILING has a solution where the number of $\star$ 's is $t$. If $s_{i, j}=(x, y) \neq \star$, then set the variables in $A_{i, j-1}, A_{i, j}$, 
$B_{i-1, j}, B_{i, j}$ such that the term corresponding to $(x, y)$ is satisfied in $\phi_{i, j}$, i.e., $a_{i, j-1, x^{\prime}}=a_{i, j, x^{\prime}}$ is true if and only if $x^{\prime}=x$, and $b_{i-1, j, y^{\prime}}=b_{i, j, y^{\prime}}$ is true if and only if $y=y^{\prime}$. Observe that this assignment is well-defined: for example, if $s_{i, j}, s_{i, j+1} \neq \star$, then they assign the same values to the variables in $A_{i, j}$ (since $s_{i, j}, s_{i, j+1}$ agree in the first component). Assign values to the remaining variables arbitrarily. It is clear that if $s_{i, j} \neq \star$, then the corresponding $\phi_{i, j}$ is satisfied.

For the other direction, assume that $t$ of the $\phi_{i, j}$ 's are satisfied in an assignment. If $\phi_{i, j}$ is satisfied, then there is a pair $(x, y) \in S_{i, j}$ such that the term corresponding to $(x, y)$ is satisfied; set $s_{i, j}=(x, y)$ in this case. Let $s_{i, j}=\star$ if $\phi_{i, j}$ is not satisfied. It is easy to verify that the $s_{i, j}$ 's form a valid solution of MATRIX TILING.

In a very similar way (details omitted), we can reduce MATRIX TILING to TMIN and TMAX, hence

Theorem 3.2. If there is a $\delta>0$ such that PLANAR TMIN or PLANAR TMAX has a PTAS with running time $2^{(1 / \epsilon)^{O(1)}} \cdot n^{O(1 / \epsilon)^{1-\delta}}$, then ETH fails.

\section{Intersection graphs}

Given a set of geometric objects in the plane, the intersection graph has one vertex for each object, and two vertices are connected by an edge if and only if they have nonempty intersection. The intersection graphs of squares, rectangles, disks, segments, and other geometric objects play an important role in many applications such as facility location [28], frequency assignment [22], and map labeling [1]. The Maximum Independent Set problem for the intersection graph of unit squares has a $2^{O(1 / \epsilon)} n$ time PTAS [15]. Here we show that this PTAS is essentially optimal:

Theorem 4.1. If there is a $\delta>0$ such that MAXIMUM INDEPENDENT SET for unit squares admits a PTAS with running time $2^{(1 / \epsilon)^{O(1)}} \cdot n^{O(1 / \epsilon)^{1-\delta}}$, then ETH fails.

Proof. The proof is by an L-reduction from MATRIX TILING. We assume that the squares are open, i.e., two squares that only touch each other do not intersect. We represent each $S_{i, j}$ by a gadget $G_{i, j}$. A gadget consists of 16 blocks of squares, where each block induces a clique. Consider the 16 squares $A_{1}, \ldots, A_{16}$ of Fig. 2a, and let $\left(x_{1}, y_{1}\right), \ldots,\left(x_{16}, y_{16}\right)$ be the coordinates of the lower left corner of these squares. (These squares are not part of the set of squares that we construct, but they will be useful in the definition of the set.) Set $\epsilon:=1 /\left(4 D^{2}\right)$ and let $\iota: Z_{D} \times Z_{D} \rightarrow Z_{D^{2}}$ be an arbitrary bijection. If $(x, y) \in S_{i, j}$ and $\iota(x, y)=r$, then we add 16 squares $B_{i, r}$ $(1 \leq i \leq 16)$ to gadget $G_{i, j}$ with coordinates

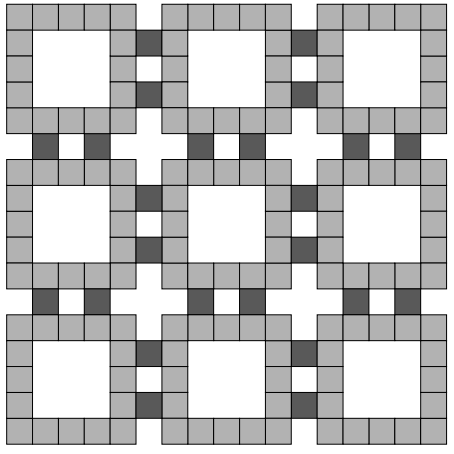

(a)

(b)

\section{Figure 2. The gadget and the way the gadgets are arranged for $k=3$ (Theorem 4.1).}

$$
\begin{array}{ll}
\left(x_{1}+r \epsilon, y_{1}+r \epsilon\right) & \left(x_{9}-r \epsilon, y_{9}-r \epsilon\right) \\
\left(x_{2}+r \epsilon, y_{2}-y \epsilon\right) & \left(x_{10}-r \epsilon, y_{10}+y \epsilon\right) \\
\left(x_{3}+r \epsilon, y_{3}-D \epsilon\right) & \left(x_{11}-r \epsilon, y_{11}+D \epsilon\right) \\
\left(x_{4}+r \epsilon, y_{4}+y \epsilon\right) & \left(x_{12}-r \epsilon, y_{12}-y \epsilon\right) \\
\left(x_{5}+r \epsilon, y_{5}-r \epsilon\right) & \left(x_{13}-r \epsilon, y_{13}+r \epsilon\right) \\
\left(x_{6}-x \epsilon, y_{6}-r \epsilon\right) & \left(x_{14}+x \epsilon, y_{14}+r \epsilon\right) \\
\left(x_{7}-D \epsilon, y_{7}-r \epsilon\right) & \left(x_{15}+D \epsilon, y_{15}+r \epsilon\right) \\
\left(x_{8}+x \epsilon, y_{8}-r \epsilon\right) & \left(x_{16}-x \epsilon, y_{16}+r \epsilon\right)
\end{array}
$$

Furthermore, we add 15 (!) squares $B_{1, D^{2}}, \ldots, B_{15, D^{2}}$ with the following coordinates:

$$
\begin{array}{ll}
\left(x_{1}+D^{2} \epsilon, y_{1}+D^{2} \epsilon\right) & \left(x_{9}-D^{2} \epsilon, y_{9}-D^{2} \epsilon\right) \\
\left(x_{2}+D^{2} \epsilon, y_{2}-D \epsilon\right) & \left(x_{10}-D^{2} \epsilon, y_{10}+D \epsilon\right) \\
\left(x_{3}+D^{2} \epsilon, y_{3}-D \epsilon\right) & \left(x_{11}-D^{2} \epsilon, y_{11}+D \epsilon\right) \\
\left(x_{4}+D^{2} \epsilon, y_{4}-D \epsilon\right) & \left(x_{12}-D^{2} \epsilon, y_{12}+D \epsilon\right) \\
\left(x_{5}+D^{2} \epsilon, y_{5}-D^{2} \epsilon\right) & \left(x_{13}-D^{2} \epsilon, y_{13}+D^{2} \epsilon\right) \\
\left(x_{6}-D \epsilon, y_{6}-D^{2} \epsilon\right) & \left(x_{14}+D \epsilon, y_{14}+D^{2} \epsilon\right) \\
\left(x_{7}-D \epsilon, y_{7}-D^{2} \epsilon\right) & \left(x_{15}+D \epsilon, y_{15}+D^{2} \epsilon\right) \\
\left(x_{8}-D \epsilon, y_{8}-D^{2} \epsilon\right) &
\end{array}
$$

It is clear that at most 16 independent squares can be selected from a gadget and an independent set of size 16 contains exactly one square $B_{i, v_{i}}$ for each $1 \leq i \leq 16$. It can be verified that for every $0 \leq j \leq D^{2}$, the squares $B_{1, j}$, $\ldots, B_{16, j}$ are independent (if they exist). Furthermore, we show that every independent set $I$ of size 16 is of this form. Observe that if $v_{i}>v_{i+1}$, then $B_{i, v_{i}}$ and $B_{i+1, v_{i+1}}$ intersect (and similarly for $B_{16, v_{16}}$ and $B_{1, v_{1}}$ ). Therefore, we get $v_{1} \leq v_{2} \leq \cdots \leq v_{16} \leq v_{1}$, which means that there is a $j$ such that $v_{i}=j$ for every $1 \leq i \leq 16$. In particular, this means that if $I$ is an independent set of size 16, then a square $B_{i, D^{2}}$ cannot appear in $I$, as there is no square $B_{16, D^{2}}$.

The $k^{2}$ gadgets are arranged as in Fig. 2b, i.e., there is an offset of 6 between the rows and columns. Adjacent gad- 
gets are connected by two blocks of additional squares as follows. If $j<k$, then in gadget $G_{i, j}$ we add the squares with coordinates $H_{r}^{1}=\left(x_{6}+1-r \epsilon, y_{6}-D^{2} \epsilon\right), H_{r}^{2}=$ $\left(x_{8}+1+r \epsilon, y_{8}+D^{2} \epsilon\right)$ for $0 \leq r<D$. These squares form the horizontal connection between $G_{i, j}$ and $G_{i, j+1}$. Similarly, if $i<k$, then we add $V_{r}^{1}=\left(x_{10}-D^{2} \epsilon, y_{10}-1+r \epsilon\right)$, $V_{r}^{2}=\left(x_{12}+D^{2} \epsilon, y_{12}-1-r \epsilon\right)$ for $0 \leq r<D$ (the vertical connection between $G_{i, j}$ and $G_{i+1, j}$ ). This completes the description of the constructed set of squares.

We claim that if the optimum of MATRIX TILING is $k^{2}-t$, then the maximum number of independent squares is $16 k^{2}+4 k(k-1)-t$. Recall that the optimum of MATRIX TILING is always at least $k^{2} / 4$, hence the reduction increases the optimum by at most a factor of $\alpha=80$. Assume that MATRIX TILING has a solution with value $k^{2}-t$. If $s_{i, j}=(x, y) \neq \star$, then select the 16 squares $B_{1, \iota(x, y)}$, $\ldots, B_{16, \iota(x, y)}$ from gadget $G_{i, j}$. If $s_{i, j}=\star$, then select the 15 squares $B_{1, D^{2}}, \ldots, B_{15, D^{2}}$ from $G_{i, j}$. Note that the $16 k^{2}-t$ squares selected so far are independent. We show that this independent set can be extended by $4 k(k-1)$ further squares by selecting two squares from each of the $2 k(k-1)$ connections. If $s_{i, j}=\star$, then the 15 squares selected from $G_{i, j}$ do not intersect any of the squares in the horizontal or vertical connections of $G_{i, j}$. Therefore, if $s_{i, j}$ or $s_{i, j+1}$ is $\star$, then it is easy to select two squares from the connection between $G_{i, j}$ and $G_{i, j+1}$. If $s_{i, j}=(x, y)$ and $s_{i, j+1}=\left(x, y^{\prime}\right)$, then we select $H_{x}^{1}$ and $H_{x}^{2}$ from the connection between $G_{i, j}$ and $G_{i, j+1}$. Observe that these two squares do not conflict with squares $B_{6, \iota(x, y)}$ and $B_{8, \iota(x, y)}$ of $G_{i, j}$ and with squares $B_{14, \iota(x, y)}$ and $B_{16, \iota(x, y)}$ of $G_{i, j+1}$. For example, $B_{6, \iota(x, y)}$ at $\left(x_{6}-x \epsilon, y_{6}-\iota(x, y) \epsilon\right)$ does not conflict with $H_{x}^{1}$ at $\left(x_{6}+1-x \epsilon, y_{6}-D^{2} \epsilon\right)$, since the difference in the first coordinate is exactly 1 . In a similar way, we can select two squares from each vertical connection, hence we obtain an independent set of size $16 k^{2}+4 k(k-1)-t$, as required.

Assume now that we have an independent set $I$ of size $16 k^{2}+4 k(k-1)-t$. If $\left|G_{i, j} \cap I\right|<16$, then set $s_{i, j}=\star$. We set $s_{i, j}$ to $\star$ also in the case if $I$ contains less than two squares from the horizontal connection between $G_{i, j}$ and $G_{i, j+1}$ or from the vertical connection between $G_{i, j}$ and $G_{i+1, j}$. For every remaining $G_{i, j}$, we have $\left|G_{i, j} \cap I\right|=16$, hence (as we have argued above), there is an $r$ such that $G_{i, j} \cap I=\left\{B_{1, r}, \ldots, B_{16, r}\right\}$. Set $s_{i, j}=\iota^{-1}(r)$ in this case. From $|I|=16 k^{2}+4 k(k-1)-t$ it follows that we set at most $t$ cells to $\star$. Assume that $s_{i, j}=(x, y)$ and $s_{i, j+1}=\left(x^{\prime}, y^{\prime}\right)$ in the constructed solution; we have to show that $x=x^{\prime}$. There are two squares $H_{x_{1}}^{1}, H_{x_{2}}^{2}$ selected from the horizontal connection between $G_{i, j}$ and $G_{i, j+1}$ (otherwise $s_{i, j}$ would be $\star$ ). Now we have that $x \geq x_{1} \geq x^{\prime}$, otherwise $H_{x_{1}}^{1}$ would intersect either $B_{6, \iota(x, y)}$ of $G_{i, j}$ or $B_{16, \iota\left(x^{\prime}, y^{\prime}\right)}$ of $G_{i, j+1}$. Similarly, by observing $H_{x_{2}}^{2}, B_{8, \iota(x, y)}$ of $G_{i, j}$, and $B_{14, \iota\left(x^{\prime}, y^{\prime}\right)}$ of $G_{i, j+1}$, we get $x \leq x_{2} \leq x^{\prime}$, hence $x=x^{\prime}$ follows. With an analogous argument, we can show that if $s_{i, j}=(x, y)$ and $s_{i+1, j}=\left(x^{\prime}, y^{\prime}\right)$, then $y=y^{\prime}$ holds.

By an argument of [24], the reduction can be made to work for unit disks as well. A similar gadget construction can be used in the case of the Minimum Dominating SET problem.

Theorem 4.2. If there is a $\delta>0$ such that MAXIMUM INDEPENDENT SET or MINIMUM DOMINATING SET for unit squares or unit disks has a PTAS with running time $2^{(1 / \epsilon)^{O(1)}} \cdot n^{O(1 / \epsilon)^{1-\delta}}$, then ETH fails.

There is a $2^{O\left(1 / \epsilon^{2}\right)} n$ time PTAS for MINIMUM VERTEX COVER on unit disk graphs [24, 27], hence the ana$\log$ of Theorem 4.2 is not true for this problem. However, we can prove a lower bound with the following argument. In the case $D=2$, the reduction of Theorem 4.1 constructs an intersection graph having bounded degee. For bounded-degree graphs, there is an L-reduction between MAXimum IndePEndent SeT and Minimum VerteX Cover. Hence by (2) of Lemma 2.8,

Theorem 4.3. If there is a $\delta>0$ such that MINIMUM VERTEX COVER for unit squares or unit disks has a PTAS with running time $2^{(1 / \epsilon)^{1-\delta}} \cdot n^{O(1)}$, then ETH fails.

Note that the lower bound of Theorem 4.3 does not match the best known approximation scheme. This might suggest that the $2^{O\left(1 / \epsilon^{2}\right)}$ PTAS of $[24,27]$ can be improved to $2^{O(1 / \epsilon)}$. We leave this as an open question.

\section{Independent Set, Vertex Cover, Dominat- ing Set}

Lipton and Tarjan [21] used the planar separator theorem of [20] to show that MAXIMUM INDEPENDENT SET on planar graphs admits a PTAS with running time $2^{O(1 / \epsilon)^{2}} n+$ $O(n \log n)$. Using a completely different approach, Baker [7] improved the dependence on $\epsilon$ to $2^{O(1 / \epsilon)} \cdot n$. Here we show that Baker's algorithm is essentially optimal:

Theorem 5.1. If there is a $\delta>0$ such that MAXIMUM INDEPENDENT SET for planar graphs has a PTAS with running time $2^{O(1 / \epsilon)^{1-\delta}} \cdot n^{O(1)}$, then ETH fails.

Proof. The proof is by presenting an L-reduction from MATRIX TILING with $D=2$ to Planar Maximum IndePENDENT SET. Each of the $k^{2}$ sets $S_{i, j}$ will be represented by an appropriate gadget. A gadget is a planar graph with distinguished vertices $a_{0}, a_{1}, b_{0}, b_{1}, c_{0}, c_{1}, d_{0}, d_{1}$ on its boundary (in this order) such that $a_{0} a_{1}, b_{0} b_{1}, c_{0} c_{1}, d_{0} d_{1}$ are edges and there are no other edges between these vertices. Let $B$ be the set of these 8 vertices. We say that a 
subset $B^{\prime} \subseteq B$ represents the pair $(x, y) \in Z_{2} \times Z_{2}$ if $B^{\prime}=\left\{a_{x}, c_{x}, b_{y}, d_{y}\right\}$. The purpose of the gadget representing $S_{i, j}$ is to ensure that the vertices selected from $B$ represent a pair from $S_{i, j}$, otherwise the gadget incurs a penalty. The following lemma states the requirements formally:

Lemma 5.2. There is a constant $c>0$ such that for every nonempty set $S \subseteq Z_{2} \times Z_{2}$, there is a gadget $G_{S}$ such that

1. The size of the maximum independent set is $\alpha\left(G_{S}\right)=$ $c+1$.

2. If $B^{\prime} \subseteq B$ represents a pair in $S$, then $B^{\prime}$ can be extended to an independent set of size $c+1$.

3. If $B^{\prime} \subseteq B$ is an independent set of size 4 , then $B^{\prime}$ can be extended to an independent set of size $c$.

4. If $I$ is an independent set of size $c+1$, then $B \cap I$ represents a set in $S$.

The proof the lemma uses standard techniques: we can argue that every set $S \subseteq Z_{2} \times Z_{2}$ can be represented by a formula, which can be turned into a planar formula, which can be turned into a planar graph using the standard reduction from 3SAT to MAXIMUM INDEPENDENT SET. Details omitted.

We construct a planar graph $G$ the following way. For every $1 \leq i, j \leq k$, we introduce a gadget $G_{i, j}$ that is the copy of $G_{S_{i, j}}$. For every $1 \leq i \leq k, 1 \leq j<k$, vertex $c_{0}$ (resp., $c_{1}$ ) of $G_{i, j}$ is connected with vertex $a_{1}$ (resp., $a_{0}$ ) of $G_{i, j+1}$; and for every $1 \leq i<k, 1 \leq j \leq k$, vertex $d_{0}$ (resp., $d_{1}$ ) of $G_{i, j}$ is connected with vertex $b_{1}$ (resp., $b_{0}$ ) of $G_{i+1, j}$.

We claim that if the optimum of MATRIX TILING is $k^{2}-t$, then $\alpha(G)=(c+1) k^{2}-t$. Assume that there is a solution of MATRIX TILING with value $k^{2}-t$. For each $s_{i, j} \neq \star$, let us select a subset $B^{\prime} \subseteq B$ that represents $s_{i, j}$, and extend it to an independent set of size $c+1$ (Prop. 3 of Lemma 5.2). Observe that the independent sets selected from neighboring gadgets do not conflict. For example, if $s_{i, j}, s_{i, j+1} \neq \star$, then they agree in the first component, hence either $c_{0}$ is selected from $G_{i, j}$ and $a_{0}$ is selected from $G_{i, j+1}$, or $c_{1}$ is selected from $G_{i, j}$ and $a_{1}$ is selected from $G_{i, j+1}$. Consider the cells with $s_{i, j}=\star$ in arbitrary order, and in each gadget, select 4 vertices of $B$ that do not conflict with the vertices already selected from the neighboring gadgets (this can be done, since it is not possible that, say, both $a_{0}$ and $a_{1}$ have a selected neighbor). Extend these 4 vertices into an independent set of size $c$ (Prop. 2 of Lemma 5.2). If $t$ is the number of $\star$ 's in the solution of MATRIX TILING, then we obtain an independent set of size $c t+(c+1)\left(k^{2}-t\right)$, hence $\alpha(G) \geq(c+1) k^{2}-t$ follows.

For the other direction, suppose that $G$ has an independent set $I$ of size $(c+1) k^{2}-t$. Since $\left|I \cap G_{i, j}\right| \leq c+1$ (Prop. 1 of Lemma 5.2), $\left|G_{i, j} \cap I\right| \leq c$ for at most $t$ of the $G_{i, j}$ 's. If $\left|G_{i, j} \cap I\right| \leq c$, then set $s_{i, j}=\star$. If $\left|G_{i, j} \cap I\right|=c+1$, then by Prop. 4 of Lemma 5.2, $B \cap I$ in $G_{i, j}$ represents a pair $(x, y)$ from $S_{i, j}$; in this case, set $s_{i, j}=(x, y)$. We can verify that this is a valid solution of MATRiX Tiling. For example, if $s_{i, j}=\left(x_{1}, y_{1}\right)$ and $s_{i, j+1}=\left(x_{2}, y_{2}\right)$, then $x_{1}=x_{2}$ : otherwise $c_{x_{1}} \in I$ of $G_{i, j}$ and $a_{x_{2}} \in I$ of $G_{i, j+1}$ would be neighbors. As the number of $\star$ 's is $t$, we get that the optimum of MATRIX TILING is at least $k^{2}-t$.

Observe that the reduction constructs bounded-degree graphs. For bounded degree graphs, there is an L-reduction from MAXimum IndePEndent Set to Minimum VeRTEX COVER. Furthermore, there is an L-reduction from Minimum Vertex Cover to Minimum Dominating SET, hence we have

Theorem 5.3. If there is a $\delta>0$ such that MINIMUM VERTEX COVER or Minimum Dominating SET for planar graphs has a PTAS with running time $2^{O(1 / \epsilon)^{1-\delta}} \cdot n^{O(1)}$, then ETH fails.

\section{TSP on planar graphs}

The Traveling Salesperson Problem (TSP) is one of the most studied optimization problems. Here we consider the special case where the distance metric is the shortest-path metric of an unweighted planar graph. That is, given a planar graph, the task is to find a tour (closed walk) of minimum length that visits every vertex at least once. The first PTAS for the problem was given by Grigni et al. [14], with running time $n^{O(1 / \epsilon)}$. Recently, this was improved to $2^{O(1 / \epsilon)} n$ by Klein [19], which is essentially optimal:

Theorem 6.1. If there is a $\delta>0$ such that TSP on unweighted planar graphs has a PTAS with running time $2^{O(1 / \epsilon)^{1-\delta}} \cdot n^{O(1)}$, then ETH fails.

Proof. The proof is by presenting an L-reduction from MATRIX TILING with $D=2$ to the problem. We say that a tour is efficient if after removing any loop (i.e., a subtour that starts and ends on the same vertex) there is at least one vertex that is not visited by the resulting shorter tour. Clearly, the optimum tour is efficient. The purpose of the exclusiveor line (Fig. 3) is to force the tour to use exactly one of the two edges $e_{1}$ and $e_{2}$. Assume that in a graph $G$ edges $e_{1}$ and $e_{2}$ are connected as in Fig. 3b, and there is an efficient TSP tour $T$ that visits each of the 16 internal vertices of the exclusive-or line exactly once. It can be verified by inspection that tour $T$ enters the exclusive-or line only once, and it either uses both $e_{1}^{\prime}$ and $e_{1}^{\prime \prime}$ (Fig. 3c), or both $e_{2}^{\prime}$ and $e_{2}^{\prime \prime}$ (Fig. 3d). In fact, we can make the assumption slightly weaker: the same conclusion holds even if we only assume that 15 of the 16 internal vertices are visited exactly once. 


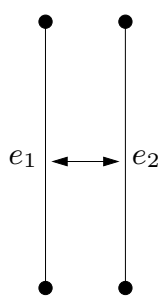

(a)

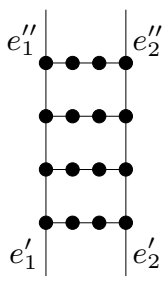

(b)

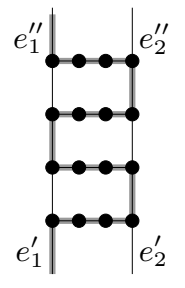

(c)

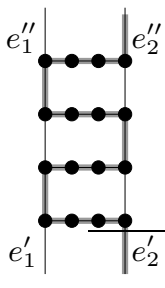

(d)
Figure 3. The exclusive-or line.

(Here we use the assumption that $T$ is efficient: otherwise it would be possible that $T$ visits the exclusive-or line twice, the first time visiting every vertex and the second time visiting only one vertex.)

We represent each set $S_{i, j}$ of MATRIX TILING by a gadget $G_{i, j}$. The gadget is a planar graph with 4 distinguished vertices $v_{1}, v_{2}, v_{3}, v_{4}$ and 4 distinguished edges $a_{0}, a_{1}, b_{0}$, $b_{1}$ on its boundary (see Fig. $4 \mathrm{a}$ ). We say that a set $\mathcal{W}$ of walks is a traversal of the gadget if both endpoints of each walk is in $\left\{v_{1}, v_{2}, v_{3}, v_{4}\right\}$ and each vertex of the gadget is visited by at least one of the walks. A traversal uses an edge $e$ if at least one of the walks go through $e$. A traversal $\mathcal{W}$ represents a pair $(x, y) \in Z_{2} \times Z_{2}$ if $a_{x}$ and $b_{y}$ are the only distinguished edges that are used by $\mathcal{W}$. The weight of a traversal is the number of vertices in the gadget that are visited more than once. The properties of the gadget are summarized in the following lemma:

Lemma 6.2. There is a constant $c>0$ such that for every nonempty set $S \subseteq Z_{2} \times Z_{2}$, there is a gadget $G_{S}$ such that

\section{There are c vertices in $G_{S}$.}

2. For every $(x, y) \in S$, there is a traversal $\mathcal{W}=$ $\left\{P_{1}, P_{2}\right\}$ of weight 0 where simple path $P_{1}$ connects $v_{1}$ and $v_{3}$, simple path $P_{2}$ connects $v_{2}$ and $v_{4}$, and $\mathcal{W}$ represents $(x, y)$.

3. For every $Z \subseteq\left\{a_{0}, a_{1}, b_{0}, b_{1}\right\}$, there is a traversal $\mathcal{W}=\left\{P_{1}, P_{2}\right\}$ of weight 1 where walk $P_{1}$ connects $v_{1}$ and $v_{3}$, walk $P_{2}$ connects $v_{2}$ and $v_{4}$, and $\mathcal{W}$ uses the edges in $Z$, but not those in $\left\{a_{0}, a_{1}, b_{0}, b_{1}\right\} \backslash Z$.

4. If a traversal $\mathcal{W}$ has weight 0 , then it represents a set $(x, y) \in S$.

The proof of this lemma is tedious, but uses only the standard gadgeteering techniques developed for proving the NP-hardness of Hamiltonian cycle. Details will appear in the full version.

Each set $S_{i, j}$ is represented by a gadget $G_{i, j}$ that is a copy of $G_{S_{i, j}}$. The gadgets are connected as shown in

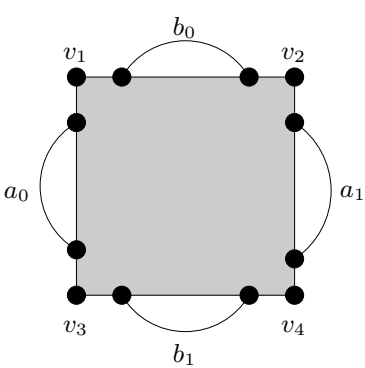

(a)

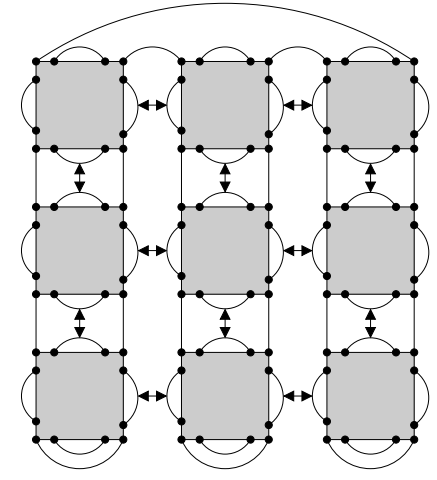

(b)

\section{Figure 4. The gadget and the way the gadgets are arranged for $k=3$ (Theorem 6.1).}

Fig. 4b. Distinguished edges of adjacent gadgets are connected with exclusive-or lines. For $1 \leq i<k, 1 \leq j \leq k$, vertex $v_{3}$ (resp., $v_{4}$ ) of $G_{i, j}$ is connected with vertex $v_{1}$ (resp., $v_{2}$ ) of $G_{i+1, j}$. Vertices $v_{3}$ and $v_{4}$ of gadget $G_{k, j}$ are connected with each other (for $1 \leq j \leq k$ ), while vertex $v_{2}$ of $G_{1, j}$ is connected with vertex $v_{1}$ of $G_{1, j+1}$ (for $1 \leq j<k)$. Finally, vertex $v_{1}$ of $G_{1,1}$ is connected with vertex $v_{2}$ of $G_{1, k}$. Observe that the number of vertices of the constructed graph is $c \cdot k^{2}+32 k(k-1)$ : there are $k^{2}$ gadgets with $c$ vertices each, and each of the $2 k(k-1)$ exclusive-or lines add 16 new vertices.

We show that if the optimum of MATRIX TILING is $k^{2}-t$, then the optimum of the constructed instance of TSP is $c \cdot k^{2}+32 k(k-1)+t$. Assume first that MATRIX TILING has a solution where the number of $\star$ 's is $t$. We construct a tour that starts at vertex $v_{1}$ of $G_{1,1}$, goes to vertex $v_{3}$ of $G_{1,1}$, goes to vertex $v_{1}$ of $G_{2,1}$, goes to vertex $v_{3}$ of $G_{2,1}$, etc., until it reaches vertex $v_{3}$ of $G_{k, 1}$. Using the edge $v_{3} v_{4}$, the tour goes to $v_{4}$ of $G_{k, 1}$ and revisits the first column by going to $v_{2}$ of $G_{k, 1}$, to $v_{4}$ of $G_{k-1,1}$, to $v_{2}$ of $G_{k-1,1}$, etc., until it reaches vertex $v_{2}$ of $G_{1,1}$. At this point the tour goes to vertex $v_{1}$ of $G_{1,2}$ and visits the second column in a similar way. Finally, when the tour reaches vertex $v_{2}$ of $G_{1, k}$, it returns to $v_{1}$ of $G_{1,1}$. Each gadget is visited twice: once by a walk from $v_{1}$ to $v_{3}$, and once by a walk from $v_{2}$ to $v_{4}$. To complete the construction of the tour, we have to find an appropriate traversal for each gadget. If $s_{i, j}=(x, y) \neq \star$, then we use the traversal of $G_{i, j}$ given by Prop. 2 of Lemma 6.2. If $s_{i, j}, s_{i, j+1} \neq \star$, then $s_{i, j}=\left(x, y_{1}\right), s_{i, j+1}=\left(x, y_{2}\right)$ implies that the traversal of $G_{i, j}$ uses edge $a_{1}$ if and only if the traversal of $G_{i, j+1}$ does not use edge $a_{0}$. Therefore, the tour can be extended to the exclusive-or lines in such a way that the tour visits the vertices of these lines exactly once (Fig. 3c or Fig. 3d). If 
$s_{i, j}=\star$, then we choose an appropriate subset of the distinguished edges, and by Prop. 3 of Lemma 6.2, we obtain a traversal of weight 1 that is compatible with the traversals of the neighboring gadgets, i.e., there is no conflict on the exclusive-or lines. If the weight of a traversal is 1 , then there is exactly one vertex in the gadget that is visited twice. Thus there are exactly $t$ vertices in the graph that are visited twice, all the other vertices are visited exactly once. Therefore, the total length of the tour is $c \cdot k^{2}+32 k(k-1)+t$.

For the other direction, assume that there is an efficient tour $T$ with length $c \cdot k^{2}+32 k(k-1)+t$. This means that there are at most $t$ vertices that are visited more than once; let $X$ be the set of these vertices. If $G_{i, j} \cap X \neq \emptyset$, then set $s_{i, j}=\star$. If at least two vertices of $X$ are in the exclusive-or line connecting $G_{i, j}$ and $G_{i, j+1}$, then set $s_{i, j}=s_{i, j+1}=\star$. The exclusive-or line connecting $G_{i, j}$ and $G_{i+1, j}$ is handled similarly. Let $G_{i, j}$ be a gadget such that $s_{i, j}$ was not set to $\star$. Each of the exclusive-or lines connected to $G_{i, j}$ contains at most one vertex of $X$, thus (as we have observed at the beginning of the proof) the line is used in a "proper way," i.e., as in Fig. $3 c$ or Fig. $3 d$. Therefore, if we remove the exclusive-or lines, then $T$ induces a traversal $\mathcal{W}$ of $G_{i, j}$ of weight 0 (since $G_{i, j} \cap X=\emptyset$ ). By Prop. 4 of Lemma 6.2, this means that $\mathcal{W}$ represents a pair $(x, y) \in S_{i, j}$; set $s_{i, j}:=(x, y)$. It easy to see that if $s_{i, j}=\left(x_{1}, y_{1}\right)$ and $s_{i, j+1}=\left(x_{2}, y_{2}\right)$, then $x_{1}=x_{2}$, otherwise it would not be true that the exclusive-or line connecting $a_{1}$ of $G_{i, j}$ and $a_{0}$ of $G_{i, j+1}$ contains at most one vertex of $X$. Similarly, if $s_{i, j}, s_{i+1, j} \neq \star$, then they agree in the second component. The number of $\star$ 's is at most the size of $X$, hence we obtain a solution with value at least $k^{2}-t$.

For the weighted version of the problem, Klein [19] gives a $2^{O\left(1 / \epsilon^{2}\right)} n$ time PTAS. We leave it as an open question whether this PTAS is optimal for the weighted version or (as in the unweighted case) it can be improved to $2^{O(1 / \epsilon)} n$ to match Theorem 6.1.

\section{References}

[1] P. K. Agarwal, M. van Kreveld, and S. Suri. Label placement by maximum independent set in rectangles. Comput. Geom., 11(3-4):209-218, 1998.

[2] J. Alber, H. Fernau, and R. Niedermeier. Parameterized complexity: exponential speed-up for planar graph problems. J. Algorithms, 52(1):26-56, 2004.

[3] J. Alber and J. Fiala. Geometric separation and exact solutions for the parameterized independent set problem on disk graphs. J. Algorithms, 52(2):134-151, 2004.

[4] A. Andoni, P. Indyk, and M. Patrascu. On the optimality of the dimensionality reduction method. In FOCS '06: Proceedings of the 47th Annual IEEE Symposium on Foundations of Computer Science (FOCS'06), pages 449-458, Washington, DC, USA, 2006. IEEE Computer Society.
[5] S. Arora. Polynomial time approximation schemes for Euclidean TSP and other geometric problems. In FOCS 1996, pages 2-11. IEEE Comput. Soc. Press, 1996.

[6] S. Arora. Polynomial time approximation schemes for Euclidean traveling salesman and other geometric problems. $J$. ACM, 45(5):753-782, 1998.

[7] B. S. Baker. Approximation algorithms for NP-complete problems on planar graphs. J. ACM, 41(1):153-180, 1994.

[8] C. Bazgan. Schémas d'approximation et complexité paramétrée. Technical report, Université Paris Sud, 1995.

[9] L. Cai, M. Fellows, D. Juedes, and F. Rosamond. The complexity of polynomial-time approximation, 2006. To appear in Theory of Computing Systems.

[10] M. Cesati and L. Trevisan. On the efficiency of polynomial time approximation schemes. Inform. Process. Lett., 64(4):165-171, 1997.

[11] J. Chen, X. Huang, I. A. Kanj, and G. Xia. Linear FPT reductions and computational lower bounds. In STOC 2004, pages 212-221, New York, 2004. ACM.

[12] I. Dinur. The PCP theorem by gap amplification. In STOC 2006, pages 241-250, 2006.

[13] R. G. Downey. Parameterized complexity for the skeptic. In Proceedings of the 18th IEEE Annual Conference on Computational Complexity, pages 147-169, 2003.

[14] M. Grigni, E. Koutsoupias, and C. Papadimitriou. An approximation scheme for planar graph TSP. In FOCS 1995, pages 640-645. IEEE, Los Alamitos, CA, 1995.

[15] H. B. Hunt, III, M. V. Marathe, V. Radhakrishnan, S. S. Ravi, D. J. Rosenkrantz, and R. E. Stearns. NC-approximation schemes for NP- and PSPACE-hard problems for geometric graphs. J. Algorithms, 26(2):238-274, 1998.

[16] R. Impagliazzo, R. Paturi, and F. Zane. Which problems have strongly exponential complexity? J. Comput. System Sci., 63(4):512-530, 2001.

[17] S. Khanna and R. Motwani. Towards a syntactic characterization of PTAS. In STOC 1996, pages 329-337.

[18] S. Khanna, M. Sudan, L. Trevisan, and D. P. Williamson. The approximability of constraint satisfaction problems. SIAM J. Comput., 30(6):1863-1920, 2001.

[19] P. Klein. A linear-time approximation scheme for planar weighted TSP. In FOCS 2005, pages 647-656. IEEE, 2005.

[20] R. J. Lipton and R. E. Tarjan. A separator theorem for planar graphs. SIAM J. Appl. Math., 36(2):177-189, 1979.

[21] R. J. Lipton and R. E. Tarjan. Applications of a planar separator theorem. SIAM J. Comput., 9(3):615-627, 1980.

[22] E. Malesińska. Graph-Theoretical Models for Frequency Assignment Problems. PhD thesis, TU Berlin, 1997.

[23] D. Marx. The closest substring problem with small distances. In FOCS 2005, pages 63-72, 2005.

[24] D. Marx. Efficient approximation schemes for geometric problems? In ESA 2005, pages 448-459, 2005.

[25] D. Marx. Parameterized complexity and approximation algorithms, 2006. To appear in The Computer Journal.

[26] S. B. Rao and W. D. Smith. Approximating geometrical graphs via "spanners" and "banyans". In STOC '98 (Dallas, TX), pages 540-550. ACM, New York, 1999.

[27] E. J. van Leeuwen. Better approximation schemes for disk graphs. In SWAT 2006, pages 316-327, 2006.

[28] D. W. Wang and Y.-S. Kuo. A study on two geometric location problems. Inform. Process. Lett., 28(6):281-286, 1988. 